\title{
Stomach Pain
}

National Cancer Institute

\section{Source}

National Cancer Institute. Stomach Pain. NCI Thesaurus. Code C78630.

A painful sensation in the stomach. 